\title{
Can research be project managed?
}

\author{
Chris J. Brown \\ Graduate School of Business, University of Stellenbosch, P.O. Box 610. Bellville 7535. South Africa
}

Received July 1999

\begin{abstract}
Due to increasing pressures on their available resources, research institutions are generally speaking in dire need of the more efficient goal achievement apparently afforded by the project management process. if applied appropriately. This article reports on an investigation in a large research organization in South Africa, to determine how closer conformance can be achieved, of research projects with the typical characteristics of 'conventional' projects so as to enhance the application of project management techniques to research work. Firstly an overview of 'conventional' project management theory is given to establish a point of departure. Thereafter a number of factors that were identified in the study, which generally inhibits project management application in functionally structured organizations, are discussed. This is followed by a discussion of certain peculiarities of research work that may cause the failure of a proper project management approach. By comparing the above with the determinants of successful project management, five groundrules are then formulated for applying project management to research environments. These groundrules are the necessary prerequisites for the framework for a generalized approach to the application of project management to research environments, which is presented in conclusion.
\end{abstract}

\section{Introduction}

Project management is heralded as the supreme management technique for the achievement of complex one-off goals within specified performance, time and cost targets. From the plethora of available literature on project management it may appear to the layman that applying project management is a simple matter of defining goals, identifying activities and resources, assembling teams and appointing authoritative project leaders. Superficially it would appear as if this notion is compatible with the management of research projects. The question to be answered is, "can research be managed (administrated) in such a structured way?'

The purpose of this article is therefore fourfold: Firstly to briefly overview relevant 'conventional' project management theory to establish an authentic point of departure.

Secondly to expound the factors that were found to inhibit project management application to functionally organized institutions generally.

Thirdly to discuss the peculiarities of research that were established, that could cause project management to fail as a research management tool.

Fourthly to expound the ground rules inferred from the above as well as a framework for a generalized approach to the application of project management to research environments.

The article is based on a comprehensive literature study, practical experience over three decades, as well as a case study of a fairly large ( $c a 4000$ employees) South African agricultural research organization presently contemplating the development of project management structures.

The author was engaged in project management education and training of $c a 500$ researchers and technicians of this organization. During and after the training programmes (20 in total spread out over the country) interviews were conducted, the data and findings of which forms the basis of this article.

\section{An overview of project management}

Project management is defined as, 'the set of techniques enabling the integrated management of the scope, time, cost and human relations frameworks pertaining to a project, to achieve the goals of the project' (Brown, 1997b)

Badiru (1996: 76) considers it to be a process of allocating and timing of resources to achieve objectives in an efficient and expedient manner. He further states that these are the same results faced by all researchers! The generic nature of the project management process can be derived from the definition, namely to manage:

- what is to be achieved?

- who is going to do it?

- how much time is needed? and

- how much money is involved?

Careful consideration of the work of researchers reveals the same responsibilities (Badiru, 1996: 77-79).

The usefulness of the systems approach for analyzing projects (Kerzner, 1994) steered the project management process towards systems in the natural sciences which lend themselves exceptionally well to the above analysis. However, historically it excluded from the project management way of goal achievement, all other types of systems that do not conform easily to the above requirements. This is especially true of biological, medical, agricultural, human and social sciences.

Comparing project-orientated management with functionally orientated management also highlights the nature of project management, for example,

Project management is: Functional management is: horizontally orientated goal driven process orientated problem orientated flexible

a finite process

applying variable resources

in indirect control of resources vertically orientated

function driven

product orientated

production orientated

stable

a continuous process

applying fixed resources

in direct control of resources 


\section{Factors that inhibit project management application in functionally structured organizations}

It is the experience of the author that due to the specialized scientific education of researchers, it is probably safe to assume that virtually all research organizations are organized into specialized functional departments. According to Graham (1994: 705) various factors that inhibit the application of project management principles are always present to a greater or lesser extent in all functionally orientated organizations. The following have been identified in this study:

1. Resistances to change within the organization because, as also stated by Krüger \& Steyn (1995: 50), project structures, leadership styles, and information, planning and control systems are departures from traditional management.

2. The projects of the organization are not of a singular nature, but form a continuum from simple to complex. This implies a continuum of managerial approaches, project organization styles and structures, which make the application of a uniform project management approach very difficult, if not impossible. See also Brown (1997a); Ford \& Randolph (1992: 271, 272, 282); and Sawle (1994: 8).

3. Top management resisted the change to project management because additional management overhead is created as well as additional staff, mainly administrative, being required. This is also experienced by Kerzner (1994) and Ford \& Randolph (1992: 276).

4. Absence of a team culture which means failure to work together with and/or to take instructions from people outside a person's own functional division. This was particularly serious with the high level researchers and conforms to the opinion of Ford et al. (1992: 282) who found a team culture to be extremely difficult to cultivate amongst highly skilled people in a rigid bureaucracy.

5. A generally low cost and time sensitivity in the organization. Project management is by definition concerned with performance, time and cost efficiency and consequently orientation thereto is a critical success factor according to Kerzner (1994); Rider (1997) and Toney (1997).

6. The de facto organization structure was found to impede on the project management approach. Organizations that are structured functionally do not provide for or even facilitate the organizing or management of activities outside the jurisdiction of a function or department. According to Ford et al. (1992: 284), however, the prime characteristic of successful cross-functional teams is indeed that they allow their members free and equal access to all communication, also outside of their own functional department.

7. Several aspects of financial management impeded on the effectiveness of project management functioning. Firstly there were insufficient incentives. See Graham (1994: 706, 707). Secondly zero-based annual departmental budgeting instead of continuous multi-year project budgeting. Thirdly the conventional financial management systems that did not include autonomous activity- and outputorientated cost management, without which project cost management is not possible (Brown, 1997a).
8. Various statutory constraints were encountered like auditory requirements, government regulations on grants and subsidies, appointment of personnel and the actions of unions.

\section{Peculiarities of research work and why project man- agement could possibly fail}

Three sets of characteristics peculiar to research work that could lead to the failure of project management were uncovered in this study. These were the characteristics of the research process, the characteristics of researchers and the characteristics of research leaders (project managers).

\section{Characteristics of the research process}

It was found a common phenomenon for the researchers to display earnest concern for the end product, but very little concern for the process of their research projects. Consequently they tend to strive to the ultimate results regardless of the efficiency of the process or of the application of the resources. They also proved to be sensitive to norming of the results (the scientific validity of the results), but not of the process (the time or money spent to achieve those results). In fact they were outright hostile to the latter. This can be attributed to a perceived 'threat to the intellectual freedom' of the researchers. Without exception they considered that of necessity the results or end products of a research endeavour are to be attained to the highest possible level of certainty in stead of to an optimum level. This is because researchers are generally evaluated on the scientific merit and integrity of their results.

The research process is by nature at a low level of certainty of inter alia,

- the input information;

- the design of the research process;

- the output (product) to be achieved during all phases of the project life cycle; and

- the resources that will be required.

Furthermore the planning norms for, and the logical workflow of the proposed activities of a research project are not always transferable from previous projects (past experiences or databases not fully applicable), nor are they known sufficiently in advance (both process and results are to be developed along the way).

A limitation in the applicability of the 'conventional' project management process is thus evident. From the definition of project management can be inferred that the basic project management techniques work well for systems where the breakdown structure identifies well-defined, discrete work packages; and where the concomitant techniques, resources and information and the end result can be forecasted to a sufficiently high level of certainty, that is for known products. A question that can now be posed is: "Under what circumstances, if any, will research projects conform to these requirements so as to enable them to be project managed?'

\section{Characteristics of the researchers}

From the investigation the following set of characteristics of the researchers, representing their 'research culture', proved to be contradictions to the requirements for project management: 
- the researchers tended to be driven by loyalty to their primary function, that is to first and foremost promote their professional skill parochially in stead of looking at the 'broad picture';

- the researchers displayed a 'prima donna' propensity which can mean being untouchable, inflexible and not inclined to share with others;

- the above invariably resulted in a low team orientation;

- the researchers displayed respect for superior knowledge and would voluntarily accept leadership of such persons easily, to the detriment of team leadership in the conventional sense; and

- they mostly displayed a science or technology orientation without sufficient management inclination or acumen. In fact most of the researchers considered (if at all) management as secondary or even a 'soft option'. This is also confirmed by other studies (Sapienza, 1995: x).

It can therefore be said that generally the organizational culture of researchers is not compatible with that required for the project way of goal achievement. Unless a reorientation of researchers is undertaken, project management will not succeed as a technique for the management of research endeavors.

\section{Characteristics of research leaders}

According to Sapienza (1995: v) in order to manage scientists well a research leader should be capable of achieving and maintaining an enthusiastic, energetic and creative group of scientists, as well as administrating (in the traditional sense) the R\&D organization effectively. That this brief could be profoundly more difficult than that for the conventional project manager is evident from the following remarks by Sapienza:

'Managing scientists entails leading people whose primary activity occurs between their ears ... the purpose (of which) ... is to generate knowledge and ideas, an endeavor that, in comparison with other organized activities, is oblique, hard to predict, unwieldy to measure and difficult to judge except in hindsight. (Therefore) .... much of ... conventional administration, ... may not be directly applicable to R\&D',

and further

'scientists are essentially trained to be solo contributors. That is why multidisciplinary teamwork and cross-functional communication and collaboration are not easily achieved within the R\&D organization' (1995: v, vi).

The important question to be answered here is, "can the work of a scientific leader, that is the management of a scientific research project, be exercised separately from that of a scientific researcher, that is pursuing the science?' About this aspect, which generated much controversy in the organization under investigation Sapienza (1995: 48), comments: 'I believe very strongly that, to be good at a job like this, you have to stay very close to the science'.

What has been discussed above means that the widely held believe, that anybody with the necessary attributes can be a project manager of any project, is in the case of research simply not true. Furthermore, that a proficient leader of a research project must be a proficient scientist (to retain the respect of the team and preserve the scientific integrity of the endeavour) and be a proficient manager (to pursue effectiveness and efficiency); in all probability a very scarce commod. ity.

The dissemination of research results and/or achievements through publication in scientific journals is mostly the primary measure of success of researchers (Terblanche, 1996). The intense awareness of the prime influence of this criterion on their careers is arguably one of the most serious impediments to a proper project management approach by the researchers. The reason is that it implies a 'results at all costs' propensity, in sharp contrast to the results/time/cost trade-off propensity of project management, to the point of being the corner stone in the organizational culture. By implication not only the top managements of research institutions, but possibly also the (entire) research fraternity face a complete rethink on the evaluation of researchers to also include the generic project management attributes. To this end Graham (1994: 706) remarks: 'if project work is not part of the performance appraisal system of researchers, project management will not be taken seriously'.

\section{Some groundrules for the application of project management to functionally orientated environ- ments}

By comparing the impediments discussed above with the literature describing the success factors for managing by project, five basic groundrules can be extracted for project management implementation in previously functionally structured organizations.

The first groundrule requires that a mind shift to the project way of management be made. The latter brings about a network of relationships, linking the various necessary inputs from different functions that is temporarily superimposed (overlaid) on the functional structure for each project. to deal with the organizational, co-ordinational and integrational complexities (Ford et al, 1992: 271). This has several consequences for an organization:

- For the project the organization's functional hierarchy becomes irrelevant. Persons from any hierarchical position may be drawn into the project team under the direction of the project leader. The latter in turn may occupy a hierarchical position subordinate to that of some of the team members. A completely different organizational culture is clearly in the offing that invariably constitutes a very complex challenge.

- For the team members responsibilities, information flow and instruction taking both transfunctionally towards the project leader and intrafunctionally towards the functional head results, which need to be spelled out very clearly (Cleland, 1990: 256).

- The specific skill input of a team member now becomes subordinate to and in support of the achievement of the project goal, and not a goal in own right. Thus, again, a new culture.

In the second instance the functional heads deem themselves threatened by the project approach because, 'the adjustment will reduce the functional manager's ability to control subordinates' activities' (Tettemer, 1991). The functional head may also view the project manager as an obstacle 
to gain favourable exposure to top management and therefore a threat to future promotion. With such perceptions co-operation by line managers will obviously be impeded. The second groundrule is therefore that the sensitivity of these new relationships cannot be overemphasized and need be carefully planned and discussed, before implementing project management (Easton \& Day, 1991). The functional heads must be reorientated towards the tasks of the creation, building and maintaining of functional capacity and excellence, instead of managing its application. For them a shift from 'generalizing' towards 'specializing' takes place. They should also be made aware of the fact that they are now relieved of many cumbersome (for them) organizational and administrative tasks.

Thirdly the project leaders feel threatened because they are taken out of their functional skills and thrusted into new responsibilities, for which they are invariably not adequately trained and which seldom carries the security of a career (Ford et al., 1992: 285). The third groundrule means that organizations must make it worth the effort for functionaries to become project managers, inclusive of status, merit assessments, evaluation measures, awards and career paths.

The fourth groundrule requires that appropriate autonomy and authority be bestowed on the project managers to enable efficient decision making regarding all the project management attributes (Brown, 1997a).

For the fifth groundrule Tettemer (1991) advocates that great emphasis be placed on comforting top administrators while displacing traditional relationships and practices with new ones. This comforting should not only be about the validity of the outcomes of the projects, but above all with the validity of the project management process.

\section{A framework for a generalised approach to project managing research endeavours}

This section contains some specific recommendations for the treatment of research work to conform to the requirements for an application of project management principles and techniques. It can, however, never be overemphasized that all of what have been discussed so far constitute very necessary preparatory actions. A dominant finding of this research was that the importance of attending to all of the factors discussed above, which might be construed as hindrances to the project management principles, usually outstrip that of the actual project managing efforts. This implies that organizations should not tread this path without a well-developed strategy and organization-fitted game plan. In this regard see also Brown (1997a).

Cukor (1992: 37) contends that projects most beneficial to promoters, were well organized, had clearly defined deliverables and had objectives related to the transfer of technology.

'It is therefore a management imperative to impose accountability, organization and discipline on research interactions. A well organized and disciplined project should not only meet deliverables but should also be accountable for time and resources'.
Bishop (1992: 12) seems to agree but warns that in research work project management ought not to be raised to the level of 'legislating success in R\&D'. Bishop (1992: 12, 13) proposes a three-pronged approach as follows:

1. Create a sense of urgency by segmenting individual projects into a series of tasks with defined descriptions of the work to be done, along with milestones which state the best guess of the amount of time needed to carry out the work. What is achieved hereby is: to force a researcher to plan; determine what can be done; and then to actually do what he/she said they could do.

2. Create a sense of progress by monitoring and tracking by some appropriate method, the defined tasks and measure the progress specifically. Contrary to 'conventional' project management where an absolute measurement against a deterministic norm would have taken place, this just need to reveal that the particular problem has not been satisfactorily solved (in scientific terms), or that a dead end is reached, or additional work is required, or an alternative path must be pursued. At that point replanning can take place by way of re-evaluation of the project (or program), readjustment of the parameters or retrenchment. What is achieved hereby is: keeping the researcher's attention on doing what he/she said could be done; demonstrating progress towards addressing the problem; and demonstrating a concerted, planned, co-ordinated effort to move the project (program) forward. 'It may not be solved yet, but you know more about it and have a better idea of what must be done'. To this end the following comment by Reiss (1992: 6) is appropriate: 'It is not a shame to overrun your project, but is unforgivable not to know that you are overrunning, nor why!'

3. Do not overly manage research projects. Do not attempt to overly define and constrain the project. Milestones of the same order as that for conventional projects are usually not needed for R\&D. Judgement should be preserved. In this regard Bishop (1992: 12, 13) offers the following advice: do not allow timing bars to extend for long periods without intermediate milestones; for each task write a criterion for success statement. This states simply what has to happen to have the task considered successfully accomplished. It becomes a good tool to monitor success and progress. It also forces the researcher to think about what he/she is trying to accomplish; that is what was intended to be done since the last review, what has been accomplished and what is expected to be done in the next time frame.

Rondinelli (1993: 24-27) proposed a classification of research projects to facilitate the establishment of an appropriate project management approach for each type of research endeavour. The proposed classification is as follows:

- Experimental projects.

- Pilot projects.

- Demonstration projects.

- Production projects.

In this research a set of determinants was developed that forms a continuum along which the above classification (from top to bottom) can be plotted (from left to right). This set is as follows: 
maximum the degree of uncertainty

maximum the risk profile

maximum the degree to which new, presently unknown knowledge will be created

minimum whether existing knowledge will be applied

minimum the complexity of the processes

minimum inter-disciplinary involvement

maximum the dominance of scientific input

minimum the dominance of managerial input

minimum the executive role of the project leader

These determinants can now be applied to describe the characteristics of each class of research. When compared with the continuum of managerial approaches, organization styles and structures described earlier (see also Brown, 1997a), the appropriate managerial approach can be developed for each class of research.

For clarification the various classes of research need to be expounded:

Experimental projects are those where little is known about the problem or the effective means of setting objectives. Unknowns and uncertainties affect nearly all aspects of such projects, from the definition of the problem and the feasibility of alternative interventions, to the choice of the most appropriate technologies, the appropriate types, magnitudes and combinations of resources, durations and sequencing of activities and organizational arrangements. These projects should probably be executed on moderate scale only, but their managers must have easy access to specialized inputs and resources.

These projects are to be classified on the lower (left hand) end of the continuum. Few conventional project planning techniques are appropriate but the proposals of Bishop discussed above, can be applied to great effect. Adaptation to the application of computerised networking techniques, to allow for reworking and loop back, will have to be developed.

The next class, moving to the right on the continuum, is pilot projects. The objective is not solely aimed at generation of new knowledge. In fact much knowledge has already been gained about the problem, the objectives, the methods, the resources to apply and the time scales. Dependence on specialized inputs and resources are at a considerably lower level. Pilot projects can be used to test results of experimental projects under varied, and unknown but predictable conditions. It can be utilized to adapt and modify methods, technologies and procedures that have been proven already.

Some of the project management techniques that rely on deterministic inputs and data can readily be applied to this type of project. In particular the work breakdown structure, time management within broader time scales, scope change management, quality assurance measures, cost management within broad parameters. Empowerment on co-ordination level for the project leader will suffice. Adaptation to the application of computerised networking techniques, to allow for reworking and loop back will have to be developed.

Demonstration projects form the next class, moving still further to the right on the continuum. This type of project is less risky and uncertain than experimental and pilot projects. Demonstration projects could make use of virtually all tools and techniques of project management. They are useful for projects with replicable results that require a minimum of supervision and training. Empowerment to a high co-ordination level for the project leader will be necessary.

Production projects are classified on the upper (right hand) end of the continuum. They probably evolve from the previous classes of projects. Production can clearly only takes place when the uncertainties and unknowns have been dealt with, or when a great deal is already known about the elements or potential impacts of a project. Clearly these projects can be managed with the full array of project management tools and techniques. Here empowerment of the project leader to that of an executive manager is usually required for successful and expedient execution of the project.

It should be noted that the proposed classification basically revolves around a continuum from probabilistic to deterministic. Project management enjoys increasing application with increasing level of certainty of work package determination.

\section{Conclusion}

It should be noted that what is stated in this article only suggests a framework which needs to be adapted to particular organizations and their projects. Especially when dealing with those systems that do not conform to project management requirements as readily as natural science based systems generally do.

This study has shown that success with the application of project management principles and techniques to research situations depend mainly on three actions. These are firstly to assure top management commitment to the philosophy and culture of the project way of management; secondly to reorientate the organization to a culture of not only preserving the highest integrity of scientific results, but also of concern for the processes for effectively and efficiently achieving those results; and thirdly to classify research endeavours to devise the appropriate (project) management approach to each.

In the final instance project management is concemed with achieving a designated unique result, by the integration of varied inputs in a lateral structure within planned time, cost and performance targets. On close inspection this is exactly what is required of researchers.

\section{References}

Badiru, A.B. 1996. Project management for research: a guide for engineering and science. London: Chapman \& Hall.

Bishop, C.J, 1992. Creating a sense of urgency, Research Technology Management, January-February: 12-13.

Brown, C.J. 1997a. Towards a strategy for project management implementation. Proceedings of the Australian Institute of Project Management 1997 National Conference. Gold Coast, Queensland, Australia, 9-12 November: 122-131.

Brown, C.J. 1997b. Unpublished class notes for the Programme in Project Management. Bellville: Graduate School of Business, University of Stellenbosch.

Cleland, D.I. 1990. Project management. strategic design and implementation. Pennsylvania: Tab Books. 
Cukor. P. 1992. How GTE Laboratories evaluate its university collaborations, Research Technology Management, March-April: 31-37.

Easton, J.L. \& Day. R.L. 1991. Planning for project management, PMBOK. Drexell Hill, Pennsylvania: PMI.

Ford, R.C. \& Randolph, G. 1992. Cross-functional structures: a review and integration of matrix organization and project management, Journal of Management, 18(2): 267-294.

Graham. R.J. 1994. Leading the change to project management, Proceedings of the 25th PMI annual seminar. Vancouver, Canada, 1719 October.

Kerzner, H. 1994. Project management: a systems approach to plan ning, scheduling and controlling. New York: Van Nostrand Reinhold.

Krüger, L.P. \& Steyn, G.P. 1995. The success factors in the implementation of project management in public sector works departments, Management Dynamics, 4(4): 49-68.

Nicholas, J.M. 1990. Managing business and engineering projects; concepts and implementation. Englewood Cliffs, New York:
Prentice hall.

Oosthuizen, P. 1997. Bye MBA. Paper presented to the PMISA Conference, Midrand, 17-19 November.

Reiss, G. 1992. Project management demystified. London: E\&F Spon.

Rondinelli, D.A. 1993. Development projects as policy experiments. 2nd ed. London: Routledge.

Sapienza, A.M. 1995. Managing scientists. Leadership strategies in biomedical research and development. New York: Wiley-Liss.

Sawle, W.S. 1994. Leading projects in a functional environment: The functional project manager. Proceedings of the 25th PMI annual seminar. Vancouver. Canada. 17-19 October.

Terblanche, J. President of the Agricultural Research Council. 1996. Personal communication, Pretoria

Tettemer, J.M. 1991. Keeping your bosses happy while implementing project management - a management view, $P M B O K$. Drexell Hill, Pennsylvania: PMI. 